\title{
Plataforma Web para acesso e disponibilização de dados climáticos
}

\author{
Angela Mazzonetto ${ }^{1}$, Felipe Borella ${ }^{1}$, Pablo Chitolina ${ }^{1}$, Guilherme Tochetto ${ }^{1}$, \\ Jonatas Casiraghi ${ }^{1}$, Fábio Augusto Antunes de Oliveira ${ }^{1}$, \\ Chou Sin Chan ${ }^{2}$, Willingthon Pavan ${ }^{1}$, Carlos Amaral Holbig ${ }^{1}$, \\ ${ }^{1}$ Universidade de Passo Fundo (UPF) \\ BR 285, Km 292,7 Caixa Postal 611 - 99052-900 - Passo Fundo - RS - Brasil \\ ${ }^{2}$ Centro de Previsão de Tempo e Estudos Climáticos (CPTEC) \\ Instituto Nacional de Pesquisas Espaciais (INPE) - Cachoeira Paulista, SP - Brasil \\ angelamazzonettofw@gmail.com, chou.sinchan@cptec.inpe.br, holbig@upf.br
}

\begin{abstract}
Weather and climate forecast are very important tools for decision making in several areas, for instance, in agriculture, hydrology and power generation. The combination of modern technologies and readily available environmental data provides a valuable mechanism to increase productivity and minimize climate risks. The subject of this meta-paper is the development of a toolkit to automate data requests, providing access and visualization of data generated by the weather forecast and climate models from CPTEC/INPE.
\end{abstract}

Resumo. A previsão do tempo e clima são ferramentas muito importantes para tomada de decisão em diversas áreas como, por exemplo, na agricultura, na hidrologia e na geração de energia. A combinação de tecnologias modernas e a disponibilização de dados meteorológicos fornecem um mecanismo para aumentar a produtividade e minimizar riscos relacionados ao clima. Neste artigo é descrito o desenvolvimento de uma plataforma para automatizar o processo de requisição, de disponibilização e de visualização de dados gerados pelos modelos de previsão de mudanças climáticas do CPTEC/INPE.

\section{Introdução}

Os sistemas de informações geográficas (GIS) têm obtido destaque na comunidade de geociências, pelo significativo papel que representam nas ciências da terra. Uma variedade de ferramentas foram desenvolvidas para fornecimento e manipulação de grandes quantidades de dados (Big Data) [Hey and Trefethen 2005]. Existe uma crescente demanda por serviços, conteúdos e produtos diversificados, que atendem segmentos e necessidades cada vez mais especificas. Lidar com esse fluxo de informações constantes torna-se uma tarefa difícil para as empresas e instituições, que muitas vezes precisam tomar decisões rápidas baseadas em análises de dados.

Diversos modelos climáticos globais vem sendo utilizados para gerar grandes volumes de dados de projeções de mudanças climáticas, por meio de diferentes cenários. Entre eles está o modelo Eta implantado no CPTEC/INPE desde 1996. Os dados gerados pelo Modelo Eta são armazenados em arquivos binários no servidor do CPTEC/INPE. Entretanto, grande parte dos usuários que utilizam estes dados necessitam 
destas informações em outros formatos, como de texto ou de planilhas eletrônicas, por exemplo. Entre os dados solicitados estão, principalmente, valores diários e mensais das variáveis bidimensionais (temperatura, umidade relativa, radiação solar, etc) disponibilizadas pelo modelo. Todo este processo, atualmente, é realizado de forma não automatizada, sendo necessário realizar ajustes nos programas de disponibilização dos dados de acordo com cada requisição realizada por cada usuário. Portanto, devido estes fatores, a criação de uma plataforma que tem como premissa automatizar o processo de disponibilização e visualização dos dados gerados pelo CPTEC/INPE é de extrema importância não só para agilizar o processo de requisição e disponibilização dos dados mas, também, possibilitar que mais usuários, dos mais diversos setores, possam ter um acesso fácil à estes dados de mudanças climáticas.

\section{Dados climáticos e meteorológicos dos modelos de previsão}

A principal ferramenta para estudos das mudanças climáticas são os modelos globais do sistema terrestre. Por outro lado, os impactos em diferentes setores de atividades são geralmente de caráter local. A resolução dos modelos globais, de cerca de $200 \times 200 \mathrm{~km}$, é considerada grosseira para estes estudos. Modelos regionais climáticos, com tamanhos de grade de cerca de $50 \mathrm{~km}$ a $20 \mathrm{~km}$, buscam atender esta necessidade de detalhamento. Entretanto, esse tamanho de grade ainda é considerado grosseiro para a maioria dos estudos relacionados com os recursos hídricos, em particular com aqueles relacionados com a disponibilidade hídrica para abastecimento humano e para a segurança alimentar. Assim, a principal inovação desta plataforma é o emprego de vários cenários de mudanças climáticas em alta resolução espacial. Uma segunda inovação é o emprego do modelo em altíssima resolução, cerca de $5 \mathrm{~km}$, em uma escala compatível com a tomada de decisões relacionados com o gerenciamento dos recursos hídricos, como é o caso dos mananciais de abastecimento afetados pelos conflitos no uso da água. O modelo Eta pode operar em modo não-hidrostático, enquanto que a maioria dos modelos climáticos simplificam em modo hidrostático.

O INPE, a partir de 1997, tem fornecido previsões numéricas de tempo com maior detalhamento para grande parte da América do Sul utilizando o modelo Eta. Desde 2002, o modelo passou a gerar previsões climáticas sazonais na resolução de 40 km sobre a região [Chou et al. 2005]. O modelo também foi utilizado para produzir estudos de mudanças climáticas [Pesquero et al. 2010, Chou et al. 2012, Marengo et al. 2012] e apoiar na elaboração da Segunda Comunicação Nacional do Brasil à Convenção-Quadro das Nações Unidas sobre Mudanças do Clima ${ }^{1}$. Novos cenários de emissão foram propostos pelo IPCC [IPCC 2013] e geraram novas projeções de mudanças climáticas globais. Projeções dos modelos HadGEM2-ES e MIROC5, em cenários de emissão RCP4.5 e RCP8.5, foram regionalizadas pelo modelo Eta do INPE [Chou et al. 2014]. RCP4.5 e RCP8.5 se referem aos cenários de emissão de gases do efeito estufa e derivam do inglês "Representative Concentration Pathway", correspondendo às forçantes radiativas de $4.5 \mathrm{Wm}-2$ e $8.5 \mathrm{Wm}-2$, respectivamente. Tais simulações regionalizadas apoiaram a elaboração da Terceira Comunicação Nacional ${ }^{2}$ e o estudo denominado "Brasil 2040: cenários e alternativas de adaptação à mudança do clima" da Secretaria de Assuntos Estratégicos da Presidência da República.

\footnotetext{
${ }^{1}$ Disponível em http://www.mct.gov.br/upd_blob/0215/215070.pdf.

${ }^{2}$ Disponível em https://goo.gl/Qelg2y
} 


\section{Desenvolvimento da plataforma web}

O desenvolvimento da plataforma web visa automatizar o processo de filtragem dos dados disponibilizados pelo CPTEC/INPE, integrando tecnologias de acesso aos dados e ao armazenamento dos mesmos. A plataforma tem como objetivo fornecer facilidade e respostas rápidas para o usuário e, ao mesmo tempo, eliminar a criação de scripts manuais feitos pelos técnicos do INPE, diminuindo a espera dos usuários pelos dados solicitados e liberando o técnico para desempenhar outras atividades. Portanto, para o desenvolvimento da plataforma foram necessários a escolha de ferramentas que possibilitam o armazenamento, o acesso e o processamento dos dados.

Os dados gerados pelo CPTEC/INPE possuem um conjunto de informações meteorológicas, climatológicas e geográficas. Sendo assim, o PostGIS mostrou ser uma ferramenta adequada para o armazenamento e para facilitar a manipulação dos dados. O PostGIS é uma extensão espacial gratuita e de código fonte livre para o PostgreSQL. Ele suporta dados geográficos e permite o acesso aos dados através de SQL (Structured Query Language), e possui funções preparadas que auxiliam na manipulação dos dados[Zhang and Yi 2010].

A previsão metereológica é gerada duas vezes ao dia, abrangendo o período de 11 dias. Esta previsão que abrange a América do Sul, é organizada em forma de grade, possuindo 75351 pontos. Na proposta inicial da plataforma, para cada ponto, serão armazenados dados referentes à cinco variáveis: vento, precipitação, umidade relativa, temperatura e radiação solar. Já para os dados de previsões climáticas foram calculadas 38 variáveis para um período histórico e futuro entre os anos de 1960 à 2100. Esses dados são gerados em formato binário disponibilizados manualmente. Para armazenamento no PostGIS estes dados serão convertidos em formato TIFF (Tagged Image File Format).

A visualização dos dados é feita por meio de uma aplicação web, através de mapas, gráficos e tabelas e poderá ser acessada, também, por um aplicativo para smartphone. Por meio de uma API (Application Programming Interface) é possível acessar a previsão climática com determinadas frequências (horária, diária ou mensal). Estes dados poderão ser requisitados e gerados em um formato específico, de acordo com a necessidade do usuário, como, por exemplo, em shapefile, tiff, netcdf, txt, binário, json e geojson.

A plataforma web e a API foram desenvolvidas usando Node.js, AngularJS e PostgreSQL/PostGIS. O desenvolvimento da aplicação segue os padrões de arquitetura de software MVC (Model-View-Controller). Os dados são obtidos por solicitações HTTP para uma API RESTful específica. JSON (JavaScript Object Notation) é o formato de troca de dados utilizado para a comunicação entre a aplicação, os dispositivos móveis e a API REST com o servidor. Uma representação do fluxo da API é apresentada na Figura 1

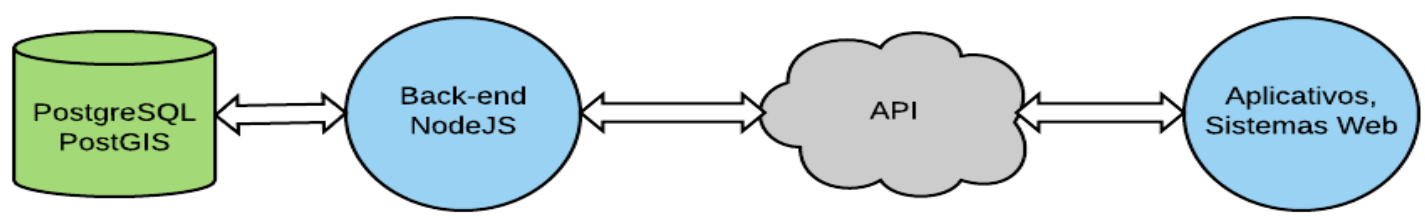

Figura 1. Fluxo da API de disponibilização de dados para os clientes (aplicativos, sistemas web, terceiros). 


\section{Conclusões}

A plataforma web aparece como uma importante ferramenta, capaz de automatizar o processo de requisição, de disponibilização e de visualização dos dados de previsão mudanças climáticas gerados pelo CPTEC/INPE, sendo capaz de filtrar os dados de acordo com os parâmetros informados e gerar o resultado no formato desejado pelos usuários de maneira intuitiva. A plataforma facilitará a disponibilização pelo CPTEC/INPE dos dados de previsão, fazendo com que eles possam ser utilizados por usuários dos mais diversos setores, auxiliando nos desafios relacionados à segurança energética e alimentar. Outra facilidade proporcionada pela plataforma é uma melhor apresentação dos dados disponibilizados, melhorando o seu entendimento e sua visualização.

\section{Agradecimentos}

Os autores agradecem ao $\mathrm{CNPq}$ pelo apoio recebido por meio do projeto "Projeções das mudanças climáticas para estudos de impactos sobre a disponibilidade hídrica no país com implicações na segurança alimentar e energética" (processo número 446197/2015-7) e da bolsa DTI-B (processo 381489/2016-7).

\section{Referências}

Chou, S. C., Bustamante, J. F., and Gomes, J. L. (2005). Evaluation of Eta model seasonal precipitation forecasts over south america, nonlin. Processes Geophys., pages 537555.

Chou, S. C., Lyra, A. A., Mourão, C., Dereczynski, C., Pilotto, I., and Gomes, J. L. (2014). Assessment of climate change over south america under RCP 4.5 and 8.5 downscaling scenarios. American Journal of Climate Change, pages 512-527.

Chou, S. C., Marengo, J. A., Lyra, A., Sueiro, G., Pesquero, J., and Alves, L. M. (2012). Downscaling of South America present climate driven by 4-member HadCM3 runs. Climate Dynamics, pages 635-653.

Hey, T. and Trefethen, A. E. (2005). Cyberinfrastructure for e-science. Science, 308(5723):817-821.

IPCC (2013). Intergovernmental Panel on Climate Change. Disponível em http: / / www. ipcc.ch/. Acesso em: 10 Fev. 2017.

Marengo, J. A., Chou, S. C., Kay, G., Alves, L. M., Pesquero, J. F., and Soares, W. R. (2012). Development of regional future climate change scenarios in south america using the Eta CPTEC/HadCM3 climate change projections: climatology and regional analyses for the amazon, São Francisco and the Paraná river basins. Climate Dynamics, pages 1829-1848.

Pesquero, J. F., Chou, S. C., Nobre, C. A., and Marengo, J. A. (2010). Climate downscaling over South America for 1961- 1970 using the Eta model. Theoretical and Applied Climatology, pages 75-93.

Zhang, L. and Yi, J. (2010). Management methods of spatial data based on PostGIS. In Circuits, Communications and System (PACCS), 2010 Second Pacific-Asia Conference on, volume 1, pages 410-413. 